\title{
Toll-Like Receptor 1/2 Stimulation Induces Elevated Interleukin-8 Secretion in Polymorphonuclear Leukocytes Isolated from Preterm and Term Newborn Infants
}

\author{
Nathan L. Thornton Mark J. Cody Christian C. Yost \\ Department of Pediatrics and Program in Human Molecular Biology and Genetics, University of Utah, \\ Salt Lake City, Utah, USA
}

\section{Key Words}

Neutrophil • Innate immunity · Toll-like receptors ·

Interleukin-8 $\cdot$ Inflammation

\begin{abstract}
Background: Neonatal neutrophil dysfunction contributes to inflammatory tissue damage in newborn infants. Toll-like receptors (TLRs) activate the innate immune response through recognition of pathogen-associated molecular patterns. Expression and function of TLRs by neonatal neutrophils has not well been characterized. Objective: We hypothesized that, compared to polymorphonuclear leukocytes (PMNs) isolated from adults, neonatal PMNs isolated from either term or preterm infants express and release different levels of inflammatory cytokines and chemokines in response to stimulation with TLR1-9 agonists. Methods: We stimulated PMNs isolated from preterm $(n=12)$ and term $(n=10)$ infants as well as adults $(n=10)$ with agonists recognized by TLRs1-9 and quantified chemokine and cytokine expression and secretion by ELISA and Luminex ${ }^{\circledR}$ multiplex quantification assay. Results: Neonatal and adult PMNs stimulated with agonists recognized by TLRs1-9 differentially secrete inflammatory products. Signaling via TLR2 heterodimers is a potent mechanism for release of in-
\end{abstract}

terleukin-8, a critical proinflammatory chemokine, by neonatal PMNs - a previously unrecognized facet of neonatal inflammation. Following TLR1/2 (PAM3CSK4) stimulation, interleukin-8 secretion by neonatal PMNs, whether term or preterm, substantially exceeds that of adult PMNs assayed in parallel. Conclusions: These studies provide new insights relevant to the inflammatory biology of neonates, both term and preterm, and implicate exaggerated PMN recruitment in neonatal syndromes of dysregulated inflammation such as necrotizing enterocolitis or neonatal chronic lung disease.

Copyright $\odot 2011$ S. Karger AG, Basel

\section{Introduction}

Polymorphonuclear leukocytes (PMNs, neutrophils) are key innate immune effector cells with critical functions in host defense and immune surveillance [1]. They respond as the first leukocyte migrating into areas of tissue injury and infection, and employ a repertoire of activities to kill bacteria [2], including phagocytosis, degranulation of antimicrobial enzymes and peptides, the generation of reactive oxygen species with antimicrobial properties, and the formation of neutrophil extracellular traps $[3,4]$. They also play an important role in tissue re-

\section{KARGER}

(C) 2011 S. Karger AG, Basel

Fax +41613061234 E-Mail karger@karger.ch www.karger.com www.karger.com/neo
Christian Con Yost, MD

Department of Pediatrics/Neonatology, University of Utah

Williams Building, 295 Chipeta Way

Salt Lake City, UT 84108 (USA)

Tel. +1 801581 7052,E-Mail christian.yost@u2m2.utah.edu 
Table 1. Neonatal patient cohort demographics

\begin{tabular}{lcc}
\hline Characteristic & Preterm $(\mathrm{n}=12)$ & Term $(\mathrm{n}=10)$ \\
\hline Gestational age (range), weeks & $28^{1} / 7\left(25^{0} / 7^{-29^{6} / 7}\right)$ & $39^{3} / 7\left(37^{1 / 7}-41^{3} / 7\right)$ \\
Birth weight (range), g & $1,125(700-1,640)$ & $3,566(2,935-4,070)$ \\
Male sex & $6(50 \%)$ & $5(50 \%)$ \\
Cesarean section delivery & $8(66 \%)$ & $2(20 \%)$ \\
5-Minute Apgar score (range) & $7.9(6-9)$ & $8.9(8-9)$ \\
Antenatal steroids given & $11(92 \%)$ & $0(0 \%)$ \\
\hline
\end{tabular}

pair and integration of the innate and adaptive immune responses [1]. If, however, these specialized, defensive responses to tissue damage or infection become dysregulated, PMNs may paradoxically become agents of inflammatory tissue injury [1, 5-10].

The developmental syndrome of neonatal neutrophil dysfunction $[4,11,12]$ not only contributes to increased risk of infection in newborn infants but may also contribute to syndromes of dysregulated inflammation observed in newborns, especially those born prematurely. Epidemiologic studies demonstrate that human infants, particularly premature babies, are at increased risk for severe infections [13-15]. Human infants are also susceptible to syndromes of dysregulated inflammation, including sepsis [15], necrotizing enterocolitis (NEC) [16], and neonatal chronic lung disease [17]. One potential mechanism that may partially account for the pronounced proinflammatory response in human neonates is exaggerated recruitment and/or activation of PMNs in affected tissues [18].

Members of the family of toll-like receptors (TLRs) serve as key activators of the innate immune response by recognizing pathogen-associated molecular patterns (PAMP) $[19,20]$. TLRs together with co-receptor molecules recognize evolutionarily conserved PAMPs expressed in microbes, and in some cases, recognize agonists generated by human cells and tissues $[19,21]$. Thus, TLRs can trigger both defensive and injurious inflammatory responses. Studies in mice suggest the possibility that intervening in immune function with selected TLR agonists may be a beneficial strategy that can reduce mortality in neonatal sepsis [22]. Nevertheless, the patterns of signaling responses mediated by TLR expression by human neonatal PMNs have not been characterized. We, therefore, used multiplex sandwich capture assays and ELISA to characterize the responses of adult and neonatal PMNs, both term and preterm, to a panel of agonists that cover the family of TLRs1-9.

TLR1/2 Stimulation in Neonatal PMNs
Table 2. Indications for preterm delivery

\begin{tabular}{ll}
\hline Indication & Preterm $(\mathrm{n}=12)$ \\
\hline Prolonged premature rupture of membranes & $6(50 \%)$ \\
Maternal chorioamnionitis & $2(17 \%)$ \\
Pregnancy-induced hypertension & $3(25 \%)$ \\
Preterm labor & $8(67 \%)$ \\
\hline
\end{tabular}

\section{Materials and Methods}

\section{Reagents}

A human TLR1-9 agonist panel was obtained from InvivoGen (www.invivogen.com). Additional reagents were purchased as follows: anti-CD15 impregnated microbeads (www.miltenyibiotec.com), IL-8 Duoset ELISA (www.rndsystems.com), Medium-199 (Lonza Biologics; M-199).

\section{PMN Isolation}

Following approval by the University of Utah IRB (Protocol Nos.: 11919, 392), we collected EDTA-anticoagulated umbilical cord blood from preterm or healthy term infants and ACD-anticoagulated peripheral blood from healthy adults (tables 1, 2). We detected no significant differences in interleukin-8 (IL-8) secretion by adult PMNs whether isolated from EDTA or ACD anticoagulated peripheral blood (data not shown). In order to obtain $>95 \%$ pure PMN sample preparations with adequate yields from preterm infant cord blood samples, we isolated the PMNs via positive magnetic immunoselection as previously described [4]. Although the potential exists for PMN activation associated with positive immunoselection, we have not found this to be the case in adult or neonatal PMNs assayed at baseline (data not shown) [23, 24]. The PMNs were resuspended and incubated in solution at a concentration of $2 \times 10^{6} / \mathrm{ml}$ in serumfree M-199.

\section{PMN Stimulation with TLR Agonists}

The following TLR agonists [21] were added to freshly isolated PMNs with final concentrations as recommended and referenced by the manufacturer (InvivoGen): PAM3CSK4 (TLR1/2 dimer, $0.5 \mu \mathrm{g} / \mathrm{ml}$ ); HKLM (TLR2, $10^{8}$ cells/ml); Poly(I:C) (TLR3, $10 \mu \mathrm{g} /$ $\mathrm{ml}$ ); Escherichia coli K12 LPS (TLR4, $5 \mu \mathrm{g} / \mathrm{ml}$ ); Salmonella ty- 


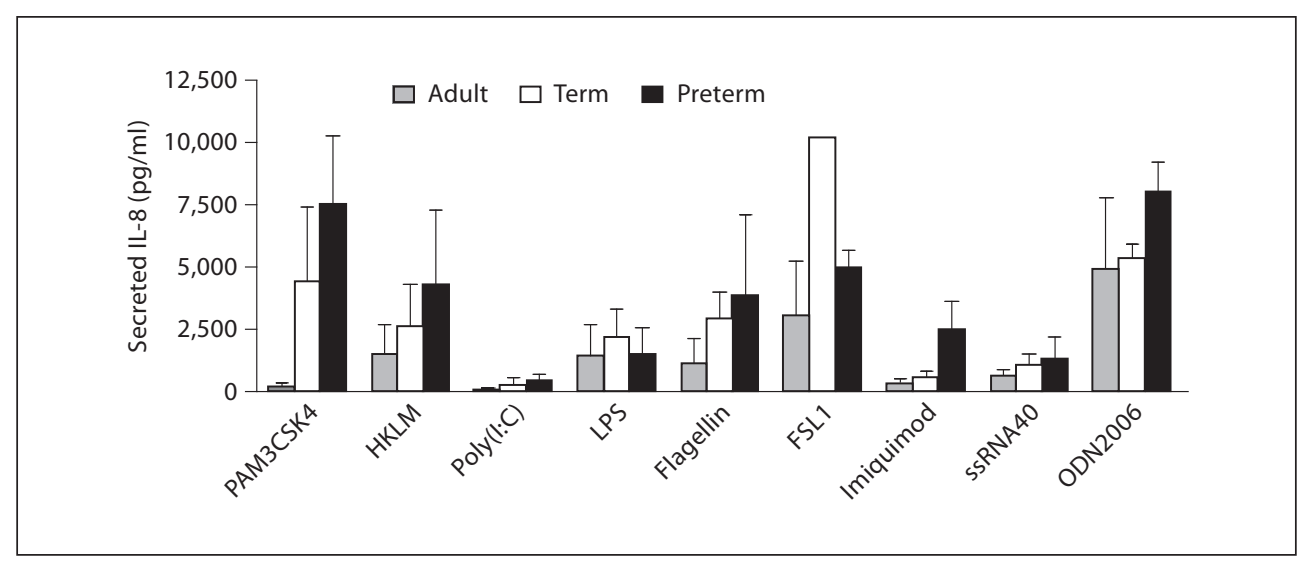

Fig. 1. Agonists recognized by TLR1-9 induce secretion of IL- 8 by adult, term, and preterm PMNs. The levels of secreted IL- 8 by human PMNs isolated from adults, term infants, and preterm infants following $8 \mathrm{~h}$ of stimulation with each of 9 TLR agonists were determined by Luminex ${ }^{\circledR}$ multiplex chemokine and cyto- kine quantification. Levels shown are mean values \pm SEM. Oneway ANOVA statistical analysis demonstrated no statistically significant differences. This figure illustrates results from 3 separate experiments with PMNs from 3 different donors in each subject group.
Table 3. Preterm neonatal cohort short-term outcomes

\begin{tabular}{lr}
\hline Outcome & $\begin{array}{r}\text { Preterm } \\
(\mathrm{n}=12)\end{array}$ \\
\hline Respiratory distress syndrome & $11(92 \%)$ \\
$\quad$ Surfactant & $8(66 \%)$ \\
$\quad$ Mechanical ventilation & $8(66 \%)$ \\
$\quad$ Ventilator days (range) & $15.6(1-58)$ \\
Chronic lung disease $\left(\mathrm{O}_{2}\right.$ at 36 weeks CGA) & $5(42 \%)$ \\
Sepsis (culture +$)$ & $5(42 \%)$ \\
Necrotizing enterocolitis ( $\geq$ stage 2) & $2(17 \%)$ \\
Retinopathy of prematurity ( $\geq$ stage 2 in zone 2) & $2(17 \%)$ \\
Intraventricular hemorrhage (PVL or $\geq$ grade 3$)$ & $1(8 \%)$ \\
Patent ductus arteriosus (medical Rx or ligation) & $4(33 \%)$ \\
\hline
\end{tabular}

phimurium Flagellin (TLR5, $2.5 \mu \mathrm{g} / \mathrm{ml}$ ); FSL-1 (TLR6/2 dimer, $0.5 \mu \mathrm{g} / \mathrm{ml}$ ); Imiquimod (TLR7, $2.5 \mu \mathrm{g} / \mathrm{ml}$ ); ssRNA40 (TLR8, $5 \mu \mathrm{g} /$ $\mathrm{ml}$ ); ODN2006 (type B; TLR9, $5 \mu \mathrm{M}$ ). PMN suspensions were then incubated at $37^{\circ} \mathrm{C}$ and $5 \% \mathrm{CO}_{2} / 95 \%$ air for an 8 -hour time course. Supernatants were collected, aliquoted, and frozen at $-20^{\circ} \mathrm{C}$.

Quantification of Chemokine and Cytokine Protein

Expression via the Luminex ${ }^{\circledR}$ Multiplex Assay

To assess the response of PMNs from all three study groups to TLR1-9 agonists, we used a multiplexed sandwich capture assay for the quantification of 13 chemokines and cytokines developed at the ARUP Institute for Experimental and Clinical Pathology at the University of Utah as previously described [25]. The chemokines or cytokines assayed included: CD40 ligand, interferon- $\gamma$, IL-10, IL-12, IL-13, IL-1 3 , IL-2, IL-2 receptor, IL-4, IL-5, IL-6, IL8 , and tumor necrosis factor- $\alpha$ (TNF- $\alpha$ ).
IL-8 ELISA

To confirm and extend the chemokine results obtained using the multiplex sandwich capture assay, quantification of IL-8 in supernatants and cell lysates was performed by IL-8 ELISA assays (R\&D Systems, Inc.) as per the manufacturer's instructions.

\section{Results}

\section{IL-8 Is a Major Product of Neonatal and Adult PMNs} following Stimulation with TLR Agonists

We studied a cohort of 12 preterm and 10 term infants with clinical data collection occurring throughout their initial hospitalization (tables 1-3). All adult PMN donors were generally healthy 18 - to 45 -year-old males or females without chronic medications with the exception of thyroid hormone supplementation.

We determined the secretion of 13 individual chemokines and cytokines by PMNs isolated from healthy adults, preterm, or term infants in response to treatment with specific agonists for each of 9 different TLRs using a Luminex ${ }^{\circledR}$ multiplex detection and quantification assay. IL-8 was, overwhelmingly, the predominant mediator of these classes secreted by PMNs stimulated with TLR agonists in this analysis (fig. 1 , table 4 , and data not shown). In parallel multiplex determinations, stimulation with agonists for each of the TLRs induced differential IL- 8 secretion by PMNs isolated from newborn infants as compared to those isolated from healthy adults (fig. 1). Although not statistically significant in these screening assays, for all of the TLR agonists, PMNs iso- 


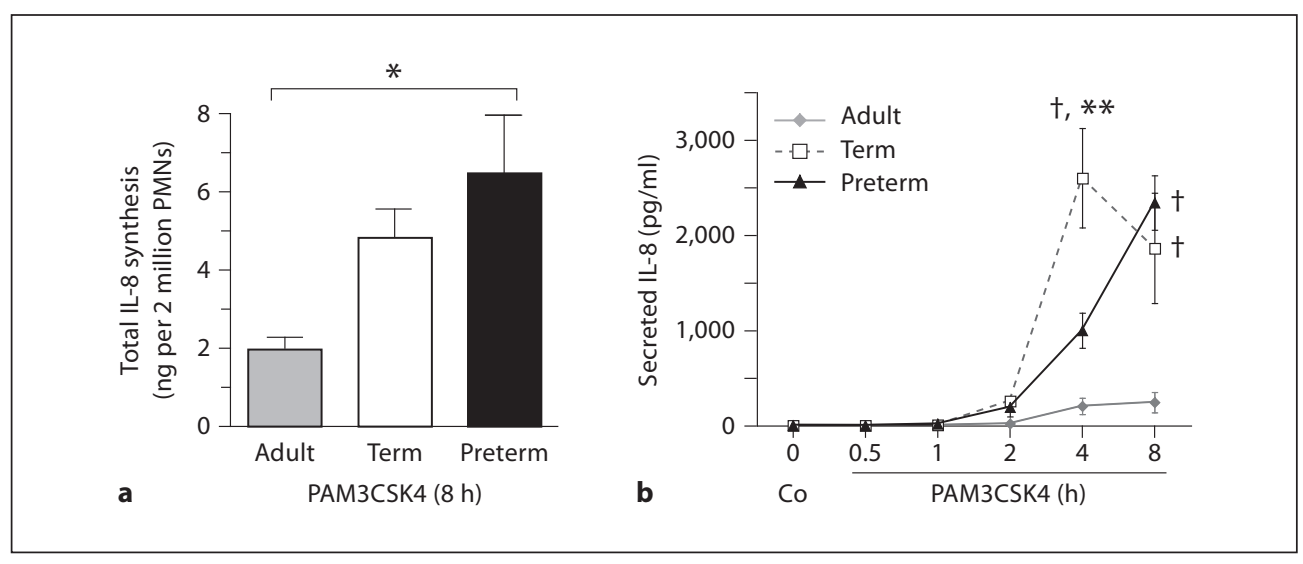

Fig. 2. Synthesis and secretion of IL- 8 by neonatal PMNs are increased following stimulation via TLR $1 / 2$ when compared to adult PMNs. a The total amount of IL- 8 synthesized by $2 \times 10^{6}$ PMNs isolated from adults and term and preterm infants following stimulation with PAM3CSK4, a TLR1/2-specific agonist, was determined by combining IL-8 concentrations from PMN cell lysates and supernatants using ELISA. Results are expressed as mean per $2 \times 10^{6} \mathrm{PMNs} \pm$ SEM. Statistical significance: ${ }^{*} \mathrm{p}<$ 0.05. The one-way ANOVA with Bonferroni's multiple comparison posttest was employed. This panel summarizes results from 4 separate experiments using PMNs from 4-6 different donors from each subject group. b Secreted concentrations of IL-8 protein were determined by ELISA following stimulation with PAM3CSK4 over an 8-hour time course. Results are expressed as mean in $\mathrm{pg} / \mathrm{ml} \pm$ SEM. Statistical significance: ${ }^{*} \mathrm{p}<0.01,4 \mathrm{~h}$ preterm vs. $4 \mathrm{~h}$ term; ${ }^{\dagger} \mathrm{p}<0.001,4$ and $8 \mathrm{~h}$ term and $8 \mathrm{~h}$ preterm vs. 4 and 8 h adult. The one-way ANOVA with Bonferroni's multiple comparison posttest was employed. This figure summarizes results from 4 separate experiments using PMNs from 4 different donors from each subject group. lated from newborn infants, whether term or preterm, secreted greater or equal amounts of IL-8 compared to PMNs isolated from adults, a result not due to differential PMN expression of TLRs 1, 2, or 4 (data not shown). Interestingly, the agonists recognized by TLR2 heterodimers (PAM3CSK4 - TLR1/2; FSL-1 - TLR2/6) induced the highest levels of secretion of the chemokine (fig. 1). We next repeated multiplex assays following treatment of PMNs from neonates and adults with an agonist specific for TLR1/2 (PAM3CSK4; table 4). Here, we found significantly elevated levels of IL-8 secreted by preterm PMNs as compared to PMNs isolated from either healthy term infants or adults, with term neonatal PMNs also secreting significantly elevated amounts of IL- 8 as compared to healthy adult PMNs. These findings confirmed our results in the focused survey of TLR agonists (fig. 1). The patterns for IL-1 $\beta$, IL-6, and TNF- $\alpha$ were different in incubations of stimulated PMNs from each subject group and, although for these screening experiments not statistically significant, suggestive of differential regulation of these products (table 4). Nevertheless, with the exception of TNF- $\alpha$ secretion by term neonatal PMNs, the levels released by neutrophils from newborn infants were greater than those released by adult PMNs (table 4). As expected, we detected very low levels of the other chemokines and cytokines - which are not generally considered PMN
Table 4. Human PMNs secrete IL-8 as a predominant inflammatory gene product following stimulation with the TLR1/2 agonist PAM3CSK4

\begin{tabular}{lccc}
\hline & Adult & Term & Preterm \\
\hline CD40L & $0.50 \pm 0.32$ & $0.70 \pm 0.23$ & $1.60 \pm 0.31$ \\
INF- $\gamma$ & $0.00 \pm 0.01$ & $0.00 \pm 0.03$ & $0.10 \pm 0.03$ \\
IL-10 & $0.00 \pm 0.00$ & $0.00 \pm 0.00$ & $0.00 \pm 0.02$ \\
IL-12 & $0.00 \pm 0.00$ & $0.10 \pm 0.06$ & $0.20 \pm 0.06$ \\
IL-13 & $0.50 \pm 0.32$ & $1.00 \pm 0.21$ & $2.10 \pm 0.44$ \\
IL-1 $\beta$ & $2.20 \pm 0.92$ & $3.20 \pm 1.86$ & $42.30 \pm 13.58$ \\
IL-2 & $0.10 \pm 0.07$ & $0.40 \pm 0.23$ & $0.60 \pm 0.14$ \\
IL-2R & $1.10 \pm 0.44$ & $1.20 \pm 0.11$ & $1.50 \pm 0.33$ \\
IL-4 & $0.00 \pm 0.00$ & $0.00 \pm 0.00$ & $0.00 \pm 0.00$ \\
IL-5 & $0.10 \pm 0.04$ & $0.10 \pm 0.10$ & $0.20 \pm 0.04$ \\
IL-6 & $0.70 \pm 0.67$ & $4.60 \pm 4.06$ & $11.00 \pm 5.95$ \\
IL-8 & $189.80 \pm$ & $4,468.30 \pm$ & $7,548.80 \pm$ \\
& 119.59 & $2,889.66^{* *}$ & $2,691.22^{*, * *}$ \\
TNF- $\alpha$ & $0.20 \pm 0.17$ & $0.10 \pm 0.05$ & $2.30 \pm 1.15$ \\
\end{tabular}

Quantification of chemokines and cytokines was performed via Luminex ${ }^{\circledR}$ multiplex analysis using PMNs stimulated with the TLR1/2 agonist PAM3CSK 4 for $8 \mathrm{~h}$. Levels shown are mean values in $\mathrm{pg} / \mathrm{ml} \pm$ SEM. Three separate experiments were performed using PMNs from 3 different donors in each subject group. Statistical significance: ${ }^{*} \mathrm{p}<0.05$ preterm vs. term; ${ }^{* *} \mathrm{p}<0.001$ preterm and term vs. adult. The one-way ANOVA with Bonferroni multiple comparisons posttest was employed. 
products - in measurements by the multiplex assay, suggesting that PMNs, and not other contaminating leukocytes, were the source of the secreted chemokines and cytokines [26] (table 4).

\section{Neonatal PMNs Synthesize and Secrete Significantly} More IL-8 following TLR1/2 Stimulation in vitro than Do PMNs Isolated from Adults

To further characterize the pattern of IL-8 synthesis and secretion by neonatal PMNs, we next quantified total and secreted IL- 8 by PAM3CSK4-stimulated neonatal or adult PMNs over an 8-hour time course measured by ELISA. We demonstrated significantly increased total IL-8 synthesis in PAM3CSK4-stimulated preterm neonatal PMNs at $8 \mathrm{~h}$ as compared to adult PMNs assayed in parallel, with a trend towards significantly increased total IL-8 synthesis in term PMNs assayed in parallel as compared to those isolated from adults (fig. 2a). Consistent with previous results in studies of adult PMNs, the baseline levels of combined IL-8 in the supernatants and cell lysates in these experiments were very low, suggesting that neither neonatal nor adult PMNs store significant amounts of pre-synthesized IL-8 [24] (data not shown). We also found significantly increased secretion of IL- 8 by term and preterm neonatal PMNs at 4 and $8 \mathrm{~h}$ as compared to PMNs isolated from healthy adults (fig. 2b). This confirmed the measurements of IL- 8 secretion by the multiplex analysis (table 4), and demonstrated the same pattern at the earlier time point in the kinetic analysis (fig. 2b). Thus, independent assays demonstrated enhanced synthesis and release of IL- 8 by neonatal PMNs activated by engagement of TLR1/2. Of interest, a similar pattern was detected in multiplex analysis when PMNs were stimulated with agonists for other TLR2 heterodimers (HKLM, FSL-1; fig. 1).

\section{Discussion}

In this report, we examined responses of adult and neonatal PMNs, both term and preterm, to a panel of agonists that cover the family of TLRs 1-9. This represents the first characterization of purified, newborn infant PMN responses to agonists for all of the TLRs with known agonists. Given the importance of neutrophil-derived chemokine and cytokine synthesis in modulation of the acute inflammatory response [26], our characterization of the 11 neutrophil-associated analytes assayed by our study represents a significant advance towards understanding the differences observed in the inflammatory states of newborn infants compared to adults. We found that IL-8, a potent neutrophil chemoattractant, is the predominant chemokine or cytokine secreted by purified human PMNs in response to TLR1-9 engagement. Furthermore, in comparison with PMNs isolated from healthy adults, PMNs isolated from human preterm and term neonates synthesize and secrete significantly increased levels of IL-8 in response to in vitro stimulation with PAM3CSK4 (table 4), an agonist for the TLR1/2 heterodimer. This issue is critical given the importance of TLR1/2 in recognition of PAMPs from a broad range of Gram-positive bacteria and other pathogens $[19,21]$.

Others have studied IL- 8 synthesis and secretion by neonatal PMNs. Zentay et al. [18] reported a similar pattern of differential increases in IL-8 secretion levels after $6 \mathrm{~h}$ of LPS stimulation in purified PMNs isolated from term infants as compared to healthy adults with even more dramatic differences noted at an 18-hour time point. While their levels of IL-8 secretion following LPS stimulation differ slightly in magnitude around the 8-hour time point (fig. 1), the pattern of increased IL-8 secretion by neonatal PMNs as compared to adult PMNs remains consistent between the two studies. More recently, Caron et al. [25] assessed cytokine and chemokine secretion in term neonatal cord blood stimulated with agonists for TLRs 1-6. While their study in whole blood differs significantly in methodology from our report of chemokine and cytokine synthesis and secretion by purified neonatal (term and preterm) and adult PMNs, their findings of significant increases in IL- $1 \beta$, IL-6, and IL-8 are consistent with neonatal PMNs contributing significantly to the circulating levels of proinflammatory mediators following TLR engagement. Levy et al. [27] recently demonstrated robust activation of antigen-presenting cells such as macrophages and dendritic cells along with increased synthesis of TNF- $\alpha$ in assays conducted in TLR8 agonist-stimulated cord blood obtained from healthy term infants. Since our results in purified neonatal and adult PMNs demonstrate very little TNF- $\alpha$ secretion by comparison at $8 \mathrm{~h}$ following PAM3CSK4 stimulation, we conclude that neutrophils contribute but minimally by comparison with antigen-presenting cells to the secretion of TNF- $\alpha$ following TLR2 engagement; however, studies evaluating PMN TNF- $\alpha$ secretion following TLR8 stimulation are warranted.

While IL-8 proved the predominant chemokine or cytokine induced by the TLR agonists studied, with the most robust IL-8 response documented for agonists engaging TLR2 heterodimers, the level of other cytokines and chemokines including IL-1 $\beta$, IL- 6 , and TNF- $\alpha$ were 
elevated in neonatal PMNs as compared to adult PMNs (table 4), although these did not reach statistical significance. Of interest, increased levels of IL-1 $\beta$ secretion by neonatal versus adult PMNs have been previously observed [28]. While these results emphasize the importance of the PMN as a potential source of cytokine synthesis and secretion, they also highlight one of the limitations of our in vitro study, that of small preterm and term infant cohort size. We anticipate that follow-up examinations using more neonatal and adult PMN samples will demonstrate significant increases in these neutrophilderived cytokines and chemokines. With this study, we were also unable to perform multiplex chemokine and cytokine analysis over an extended time course; we recognize that early increases in cytokines like TNF- $\alpha$ or IL- 6 may have been missed. Follow-up studies aimed at further characterizing PMN secretion of these important inflammatory mediators over time are planned.

Given the population of preterm infants studied, the effect of antecedent events associated with or causal of delivery at $<30$ weeks' gestation, must be recognized, but cannot be eliminated as confounding aspects of this study. Nevertheless, the response to TLR1/2 stimulation by both preterm and term neonatal PMNs with increased IL-8 secretion regardless of the antenatal inflammatory state suggests a developmental tendency towards the proinflammatory state in newborn infants as compared to adults. Along those lines, we also detected no differences in IL-8 secretion following PAM3CSK4 stimulation of PMNs isolated from infants born by cesarean section versus vaginal delivery (data not shown) consistent with previous studies [29], although a difference might have been expected given more recent reports [30, 31].

Recognition of PAMPs by TLRs expressed on PMNs with subsequent downstream signaling leads to cellular activation and de novo gene expression to aid in clearance of infectious agents and restoration of tissue homeostasis. Secretion of the chemokine IL- 8 by activated PMNs recruits other PMNs to areas of tissue damage and inflammation, thus amplifying the acute inflammatory response. Engagement of TLR4 by lipopolysaccharide also extends the life span of the activated PMNs, leading to increased PMN accumulation in areas of inflammation [32]. Given the potential for PMN-mediated tissue damage resulting from dysregulated responses to infection or tissue damage, our finding that PMNs isolated from newborn infants respond to agonists for several TLRs in addition to TLR4 with IL-8 secretion (fig. 1) is intriguing. This observation suggests that enhanced generation of this chemokine may be a key contributor to dysregulated

PMN recruitment and activation leading to inflammatory tissue damage in newborn infants associated with syndromes of dysregulated inflammation such as NEC and chronic lung disease in those born prematurely. Indeed, the short-term, clinical outcomes in this cohort of prematurely born infants, confirm that these patients are at risk for such syndromes of dysregulated inflammation (table 3). Recent data using an animal model of NEC suggest a prominent role for altered TLR signaling and cytokine elaboration in gut tissue prior to clinical manifestation of bowel necrosis [33].

\section{Acknowledgements}

We thank Drs. Guy A. Zimmerman, Andrew S. Weyrich, Estelle S. Harris, Timothy R. LaPine, and Harry R. Hill for their suggestions and helpful discussions. The ARUP Institute for Clinical and Experimental Pathology at the University of Utah provided valuable expertise in performing the multiplex detection and quantification assays. We thank the clinical staffs in the NICUs at both the University of Utah Health Sciences Center and Intermountain Medical Center for help in obtaining term and preterm infant cord blood samples. We also thank Jenny Pierce and Diana Lim for assistance with the manuscript and figure preparation and submission.

This research was supported in part by the Children's Health Research Center of the University of Utah and the Primary Children's Medical Foundation (awards to N.L. Thornton and C.C. Yost), as well as by a Physician-Scientist Training Award (K08) from the National Institutes of Health (HD-049699 C.C. Yost).

References

Neonatology 2012;101:140-146
1 Nathan C: Neutrophils and immunity: challenges and opportunities. Nat Rev Immunol 2006;6:173-182.

2 Segal AW: How neutrophils kill microbes. Annu Rev Immunol 2005;23:197-223.

-3 Brinkmann V, Reichard U, Goosmann C, Fauler B, Uhlemann Y, Weiss DS, Weinrauch Y, Zychlinsky A: Neutrophil extracellular traps kill bacteria. Science 2004;303:15321535.

-4 Yost CC, Cody MJ, Harris ES, Thornton NL, McInturff AM, Martinez ML, Chandler NB, Rodesch CK, Albertine KH, Petti CA, Weyrich AS, Zimmerman GA: Impaired neutrophil extracellular trap (net) formation: a novel innate immune deficiency of human neonates. Blood 2009;113:6419-6427.

5 Kaufmann I, Hoelzl A, Schliephake F, Hummel T, Chouker A, Peter K, Thiel M: Polymorphonuclear leukocyte dysfunction syndrome in patients with increasing sepsis severity. Shock 2006;26:254-261.

6 Aldridge AJ: Role of the neutrophil in septic shock and the adult respiratory distress syndrome. Eur J Surg 2002;168:204-214. 
7 Wipke BT, Allen PM: Essential role of neutrophils in the initiation and progression of a murine model of rheumatoid arthritis. J Immunol 2001;167:1601-1608.

$\checkmark 8$ Fiocchi C: Inflammatory bowel disease: etiology and pathogenesis. Gastroenterology 1998;115:182-205.

-9 Coxon A, Cullere X, Knight S, Sethi S, Wakelin MW, Stavrakis G, Luscinskas FW, Mayadas TN: Fc gamma RIII mediates neutrophil recruitment to immune complexes. A mechanism for neutrophil accumulation in immune-mediated inflammation. Immunity 2001;14:693-704.

-10 Nguyen CN, Schnulle PM, Chegini N, Luo X, Koenig JM: Neonatal neutrophils with prolonged survival secrete mediators associated with chronic inflammation. Neonatology 2010;98:341-347.

-11 Koenig JM, Yoder MC: Neonatal neutrophils: the good, the bad, and the ugly. Clin Perinatol 2004;31:39-51.

-12 Carr R: Neutrophil production and function in newborn infants. Br J Haematol 2000;110: 18-28.

-13 Angus DC, Linde-Zwirble WT, Lidicker J, Clermont G, Carcillo J, Pinsky MR: Epidemiology of severe sepsis in the united states: analysis of incidence, outcome, and associated costs of care. Crit Care Med 2001;29: 1303-1310.

-14 Stoll BJ, Hansen N, Fanaroff AA, Wright LL, Carlo WA, Ehrenkranz RA, Lemons JA, Donovan EF, Stark AR, Tyson JE, Oh W, Bauer CR, Korones SB, Shankaran S, Laptook AR, Stevenson DK, Papile LA, Poole WK: Changes in pathogens causing earlyonset sepsis in very-low-birth-weight infants. N Engl J Med 2002;347:240-247.
15 Stoll BJ, Hansen NI, Higgins RD, Fanaroff AA, Duara S, Goldberg R, Laptook A, Walsh M, Oh W, Hale E: Very low birth weight preterm infants with early onset neonatal sepsis: the predominance of gram-negative infections continues in the national institute of child health and human development neonatal research network, 2002-2003. Pediatr Infect Dis J 2005;24:635-639.

16 Hunter CJ, Upperman JS, Ford HR, Camerini V: Understanding the susceptibility of the premature infant to necrotizing enterocolitis (NEC). Pediatr Res 2008;63:117-123.

17 Speer CP: Pulmonary inflammation and bronchopulmonary dysplasia. J Perinatol 2006;26(suppl 1):S57-S62; discussion S63S54.

18 Zentay Z, Sharaf M, Qadir M, Drafta D, Davidson D: Mechanism for dexamethasone inhibition of neutrophil migration upon exposure to lipopolysaccharide in vitro: role of neutrophil interleukin-8 release. Pediatr Res 1999;46:406-410.

19 Takeuchi O, Akira S: Pattern recognition receptors and inflammation. Cell 2010;140: 805-820.

20 Akira S, Uematsu S, Takeuchi O: Pathogen recognition and innate immunity. Cell 2006 124:783-801.

21 Takeda K, Akira S: Toll-like receptors in innate immunity. Int Immunol 2005;17:1-14.

22 Wynn JL, Scumpia PO, Winfield RD, Delano MJ, Kelly-Scumpia K, Barker T, Ungaro R, Levy O, Moldawer LL: Defective innate immunity predisposes murine neonates to poor sepsis outcome but is reversed by TLR agonists. Blood 2008;112:1750-1758.

23 Lindemann SW, Yost CC, Denis MM, McIntyre TM, Weyrich AS, Zimmerman GA: Neutrophils alter the inflammatory milieu by signal-dependent translation of constitutive messenger RNAs. Proc Natl Acad Sci U S A 2004;101:7076-7081.

-24 Yost CC, Denis MM, Lindemann S, Rubner FJ, Marathe GK, Buerke M, McIntyre TM, Weyrich AS, Zimmerman GA: Activated polymorphonuclear leukocytes rapidly synthesize retinoic acid receptor-alpha: a mechanism for translational control of transcriptional events. J Exp Med 2004;200:671-680.
25 Caron JE, La Pine TR, Augustine NH, Martins TB, Hill HR: Multiplex analysis of tolllike receptor-stimulated neonatal cytokine response. Neonatology 2010;97:266-273.

26 Scapini P, Lapinet-Vera JA, Gasperini S, Calzetti F, Bazzoni F, Cassatella MA: The neutrophil as a cellular source of chemokines. Immunol Rev 2000;177:195-203.

\27 Levy O, Suter EE, Miller RL, Wessels MR: Unique efficacy of toll-like receptor 8 agonists in activating human neonatal antigenpresenting cells. Blood 2006;108:1284-1290.

-28 Contrino J, Krause PJ, Slover N, Kreutzer D: Elevated interleukin-1 expression in human neonatal neutrophils. Pediatr Res 1993;34: 249-252.

-29 Usmani SS, Kamran S, Harper RG, Wapnir RA, Mehta R: Effect of maternal labor and mode of delivery on polymorphonuclear leukocyte function in healthy neonates. Pediatr Res 1993;33:466-468.

$>30$ Molloy EJ, O’Neill AJ, Grantham JJ, Sheridan-Pereira M, Fitzpatrick JM, Webb DW, Watson RW: Labor promotes neonatal neutrophil survival and lipopolysaccharide responsiveness. Pediatr Res 2004;56:99-103.

31 Weinberger B, Vetrano AM, Syed K, Murthy S, Hanna N, Laskin JD, Laskin DL: Influence of labor on neonatal neutrophil apoptosis, and inflammatory activity. Pediatr Res 2007; 61:572-577.

32 Molloy EJ, O’Neill AJ, Doyle BT, Grantham JJ, Taylor CT, Sheridan-Pereira M, Fitzpatrick JM, Webb DW, Watson RW: Effects of heat shock and hypoxia on neonatal neutrophil lipopolysaccharide responses: altered apoptosis, toll-like receptor-4 and CD11B expression compared with adults. Biol Neonate 2006;90:34-39.

33 Liu Y, Zhu L, Fatheree NY, Liu X, Pacheco SE, Tatevian N, Rhoads JM: Changes in intestinal toll-like receptors and cytokines precede histological injury in a rat model of necrotizing enterocolitis. Am J Physiol Gastrointest Liver Physiol 2009;297:G442-G450. 